\title{
Radially symmetric growth of nonnecrotic tumors
}

\author{
Joachim Escher and Anca-Voichita Matioc
}

\begin{abstract}
The growth of tumors is an important subject in recent research. We present here a mathematical model for the growth of nonnecrotic tumors in all the three regimes of vascularisation. This leads to a freeboundary problem which we treat by means ODE techniques. We prove the existence of a unique radially symmetric stationary solution. It is also shown that, if the initial tumor is radially symmetric, there exists a unique radially symmetric solution of the evolution equation, which exists for all times. The asymptotic behaviour of this solution will be discussed in relation to the parameters characterizing cell proliferation and cell death.
\end{abstract}

Mathematics Subject Classification (2000). 34D20, 35K80, 35R05.

Keywords. Tumor growth, Free boundary problem, Radial symmetry.

\section{Introduction and the main results}

The study of tumor growth models is a very current topic in mathematics. During the last four decades an increasing number of mathematical models have been proposed to describe the growth of solid tumors (see $[2,6-8]$ and the literature therein). There is a three level approach in modeling the complex phenomena influencing and describing the processes inside a tumor. Models at sub-cellular level take into consideration that the evolution of a cell is determined by the genes in its nucleus, at cellular level they model cell-cell interaction, and at macroscopic level, when the tumor is considered to consist of three zones: an external proliferating zone near high concentration of nutrient, an intermediate layer, and an internal zone consisting of necrotic cells only. Very often models combine aspects from different scales. There are also a large variety of different types of models: biological models, consisting of coupled ODE systems where the variables correspond to some biological properties of an entire population; mechanical models yield to determine the cell movement based on physical forces; the discrete models handle single-cell scale 
phenomena and the effects are then examined at macroscopic scale, and moving boundary models, when the macroscopic description of biological tissues is obtained from continuum mechanics or microscopic description at cellular level.

Using algebraic manipulations Cristini et al. obtain in [5] a new mathematical formulation of an existing model (see $[4,8,11]$ ) which describes the evolution of nonnecrotic tumors in different regimes of vascularisation (see the cases (i)-(iii) presented in Sect. 2). The simpler model, as presented in $[4,8,11]$, has been studied extensively by different authors, see, e.g. $[3,6,7,9]$ and the references therein. In particular it is shown in these papers that if the parameters belong to an appropriate range, then the mathematical formulation possesses a unique radially symmetric solution. Moreover the stability properties of this solution under general perturbations as well as bifurcation phenomena are studied.

In contrast, for the more involved model presented in [5] (and which we consider here), not many analytic results are available. Before we state our main result, let us introduce the system we are interested in. As widely used in the modeling the tumor is treated as an incompressible fluid and tissue elasticity is neglected. Cell-to-cell adhesive forces are modeled by surface tension at the tumor-tissue interface. The growth of the tumor is governed by a balance between cell-mitosis and apoptosis (programed cell-death). The rate of mitosis $\left(\lambda_{M}\right)$ depends on the concentration of nutrient, and no inhibitor chemical species are present.

The two-dimensional system describing the evolution of the tumor is a fully nonlinear system consisting of two decoupled Dirichlet problems, one for the rate $\psi$ at which nutrient is added to the tumor domain $\Omega(t)$ and one for the pressure $p$ inside the tumor. These two variables are coupled by a relation which describes the dynamic of the tumor boundary. The full system reads as follows:

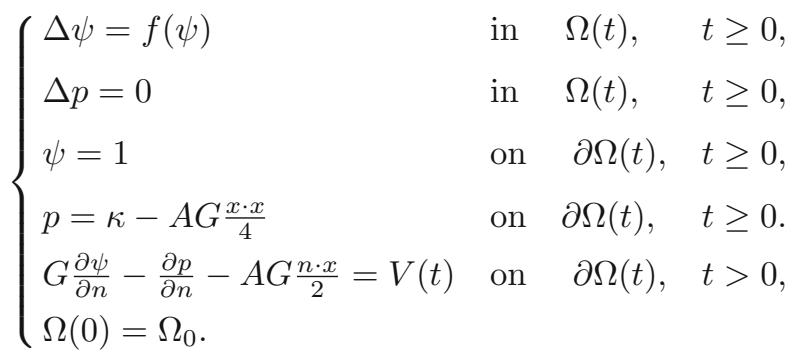

Here $\Omega_{0}$ is the initial state of the tumor, $V$ is the normal velocity of the tumor boundary, and the constants $A$ and $G$ have biological meaning (see Sect. 2 for the explicit formula). The function $f \in C^{\infty}([0, \infty))$ has the following properties

$$
f(0)=0 \quad \text { and } \quad f^{\prime}(\psi)>0 \quad \text { for } \psi \geq 0 .
$$

In [5] the special case $f(r)=r$ is considered. In this situation the first equation of the system is linear, and if the tumor domain is a sphere or an infinite 
cylinder the solution is known through an explicit formula. The general case is considerably more involved, the equation being highly nonlinear.

Our analysis shows that, under the assumptions (1.2), problem (1.1) possesses a unique radially symmetric solution iff $(A, G) \in(0, f(1)) \times(\mathbb{R} \backslash\{0\})$. The radius of this tumor, which we denote by $R_{A}$, depends only on the constant $A$. This matches also the previous results obtained for the simpler model (see $[6,8]$ ). Furthermore, given $(A, G) \in \mathbb{R}^{2}$, we show the tumor exists in the large, provided it is symmetric initially. The asymptotic behaviour of these tumors for $t \rightarrow \infty$ is described in Theorem 1.2.

If $G=0$ then any disc is a stationary solution. Therefore we consider only the case $G \neq 0$ here.

Theorem 1.1 (Steady states). Given $(A, G) \in(0, f(1)) \times(\mathbb{R} \backslash\{0\})$, there exists a unique radially symmetric, stationary solution to problem (1.1). The radius $R_{A}$ of the stationary tumor depends only on the parameter $A$ and decreases with respect to this variable.

Given $R>0$, we denote by $D(0, R)$ the disc in $\mathbb{R}^{2}$ with centre 0 and radius $R$.

Theorem 1.2 (Asymptotic behaviour). If the tumor is initially radially symmetric, i.e. $\Omega_{0}=D\left(0, R_{0}\right)$ for some $R_{0}>0$, then it remains symmetric for all times and the following assertions hold:

(a) If $(A, G) \in(0, f(1)) \times(0, \infty)$, there exist positive constants $\omega, C>0$ and $r$ such that if $\left|R_{0}-R_{A}\right| \leq r$, then

$$
\left|R(t)-R_{A}\right| \leq C e^{-\omega t} \quad \text { for } \quad t \geq 0 .
$$

(b) If $(A, G) \in[f(1), \infty) \times(0, \infty)$, we have

$$
R(t) \underset{t \rightarrow \infty}{\searrow} 0 .
$$

(c) If $(A, G) \in[f(1), \infty) \times(-\infty, 0)$, we have that

$$
R(t) \underset{t \rightarrow \infty}{\nearrow} \infty \text {. }
$$

(d) If $(A, G) \in(0, f(1)) \times(-\infty, 0)$, then the stationary solution is unstable, since

$$
R(t) \underset{t \rightarrow \infty}{\longrightarrow}\left\{\begin{array}{lll}
0, & \text { if } & R_{0}<R_{A} \\
\infty, & \text { if } & R_{0}>R_{A} .
\end{array}\right.
$$

(e) If $(A, G) \in(-\infty, 0] \times(-\infty, 0) \cup(0, \infty)$, then

$$
R(t) \underset{t \rightarrow \infty}{\longrightarrow}\left\{\begin{array}{lll}
0, & \text { if } & G<0 \\
\infty, & \text { if } & G>0 .
\end{array}\right.
$$

In all the above situations (a)-(e) the convergence is monotone. Moreover, $R(t)$ converges exponentially fast except the case $(b)$ when $f(1)=A$ and in the case $(e)$ when $A=0$ and $G>0$. 
Compared with [8], where a vascularised tumor has been studied, our model here includes also the cases of low and moderate vascularised regimes. In the low vascularisation regime it is possible that the tumor disappears if the parameter $A$ is large enough, meaning that sufficiently many cells are dying. Furthermore, also in the high-vascularised regime it is possible that the tumor vanishes, but in this case the parameter $A$ must be very small. Notice that if initially tumor growth occurs then the radius of the tumor is always increasing in time. Also, if the radius decreases at $t=0$, then the tumor is shrinking as time evolves.

The outline of the paper is as follows: We start by describing the algebraic manipulations that lead to (1.1). In Sect. 3 we transform the system describing the radially symmetric, stationary solutions of (1.1) into an equation in the variable $R$. This equation will be solved using a nice representation for the function $\psi$ (see formula (3.26)). Theorem 1.2 will be proved in Sect. 4 by making use of ODE technics.

\section{The mathematical model}

We give first a brief presentation of the modeling aspects described in $[4,8,11]$. The model there is posed in terms of conservation laws for the nutrient and tumor-cell concentration.

Let $\Omega(t)$ denote the tumor domain at time $t \geq 0$. The quasi-steady diffusion equation for the nutrient concentration $\sigma$ is

$$
0=D \cdot \Delta \sigma+\psi
$$

where $D$ is the diffusion constant and $\psi$ the rate of change of the nutrient. This rate $\psi$ incorporates all sources (in our situation only blood provides the tumor with nutrient) and sinks of nutrient in the tumor volume. Nutrient is supplied by the vasculature at a rate $\psi_{B}\left(\sigma, \sigma_{B}\right)$, where $\sigma_{B}$ is the nutrient concentration in blood. The rate of consumption of nutrient by the tumor cells is $\lambda \sigma$, where $\lambda$ is a constant. The blood-tissue transfer is assumed to be linear:

$$
\psi_{B}=-\lambda_{B}\left(\sigma-\sigma_{B}\right),
$$

where $\lambda_{B}$ is a positive constant. Thus, the rate $\psi$ is given by

$$
\psi=-\lambda_{B}\left(\sigma-\sigma_{B}\right)-\lambda \sigma .
$$

Because the tumor is treated as an incompressible fluid the velocity field $\mathbf{v}$ in $\Omega(t)$ satisfies the continuity equation:

$$
\nabla \mathbf{v}=\lambda_{P},
$$

where $\lambda_{P}$, the cell-proliferation rate, is given by the expression

$$
\lambda_{P}=b \sigma-\lambda_{A},
$$

with $b$ and $\lambda_{A}$ positive constants. The constant $\lambda_{A}$ plays an important role in our analysis because it describes the rate of apoptosis. The velocity is assumed to obey Darcy's law:

$$
\mathbf{v}=-\mu \nabla P,
$$


where $\mu$ is the cell mobility and $P$ is the pressure inside $\Omega(t)$. The boundary condition for concentration at the boundary $\partial \Omega(t)$ is given by

$$
\sigma=\sigma^{\infty}
$$

where $\sigma^{\infty}$ is the nutrient concentration outside the tumor volume, assumed to be constant. The characteristic mitosis rate is set to be $\lambda_{M}=b \sigma^{\infty}$. Pressure is assumed to satisfy the Laplace-Young boundary condition

$$
P=\gamma \kappa_{\partial \Omega(t)},
$$

where $\gamma$ is the surface tension related to cell-to-cell adhesive forces and $\kappa_{\partial \Omega(t)}$ is the curvature of the curve $\partial \Omega(t)$. The normal velocity $V=n \cdot \mathbf{v}$ at the tumor boundary $\partial \Omega(t)$ is

$$
V=-\mu n \cdot \nabla P
$$

with $n$ the outward unit normal at $\partial \Omega(t)$.

Nondimensionalisation. The relations (2.1) and (2.3) reveal that there is an intrinsic length scale

$$
L_{D}=D^{\frac{1}{2}} \cdot\left(\lambda_{B}+\lambda\right)^{-\frac{1}{2}}
$$

which for $\lambda_{B}=0$ estimates the stable size of an avascular tumor when diffusion of nutrient and consumption balance. By nondimiensionalising lengths with $L_{D}$ we obtain, from (2.6) and (2.8), an intrinsic relaxation time scale $\lambda_{R}^{-1}$, corresponding to the rate:

$$
\lambda_{R}=\mu \gamma L_{D}^{-3}
$$

associated to the relaxation mechanism, cell mobility and surface tension. This rate is used to nondimensionalise time.

We introduce now the parameters $A$ and $G$ by setting

$$
\begin{gathered}
A=\frac{\frac{\lambda_{A}}{\lambda_{M}}-B}{1-B}, \\
G=\frac{\lambda_{M}}{\lambda_{R}} \cdot(1-B) .
\end{gathered}
$$

The parameter $G$ describes the relative rate of mitosis to the relaxation mechanism (cell mobility and cell-to-cell adhesion). The parameter $A$ describes the balance between apoptosis and mitosis. Both parameters also include the effect of vascularisation $B$, which is given by

$$
B=\frac{\sigma_{B}}{\sigma^{\infty}} \frac{\lambda_{B}}{\lambda+\lambda_{B}}
$$


The parameters $A$ and $G$ allow us to subdivide the growth of the tumor into three regimes associated with increasing degrees of vascularisation:

(i) low vascularisation (diffusion dominated, e.g. in vitro cell cultures):

$$
\left.G \geq 0 \text { and } A>0 \quad \text { (i.e. } B<\lambda_{A} / \lambda_{M}\right),
$$

(ii) moderate vascularisation: $G \geq 0$ and $A \leq 0$ (i.e. $\left.1>B \geq \lambda_{A} / \lambda_{M}\right)$,

(iii) high vascularisation: $G<0$ (i.e. $B>1)$.

The moderate and high vascularisation cases correspond to the regimes observed in in vivo experiments.

Further on we introduce the modified concentration $\bar{\psi}$ and the modified pressure $\bar{p}$ so that

$$
\begin{aligned}
\sigma & =\sigma^{\infty}(1-(1-B)(1-\bar{\psi})) \\
P & =\frac{\gamma}{L_{D}}\left(\bar{p}+G(1-\bar{\psi})+A G \frac{x \cdot x}{4}\right) .
\end{aligned}
$$

The following lemma shows that the problem just stated above can be reformulated in terms of the system (1.1). Indeed, dropping down the bars in (2.16)-(2.18) yields the two-dimensional version of (1.1).

Lemma 2.1. The dimensional problem given by the relations (2.1)-(2.9) is equivalent to the following two non-dimensional decoupled problem:

$$
\left\{\begin{array}{lll}
\Delta \bar{\psi}=\bar{\psi} & \text { in } & \Omega(t) \\
\bar{\psi}=1 & \text { on } & \partial \Omega(t)
\end{array}\right.
$$

and

$$
\begin{cases}\Delta \bar{p}=0 & \text { in } \Omega(t) \\ \bar{p}=\kappa_{\partial \Omega(t)}-A G \frac{x \cdot x}{4} & \text { on } \partial \Omega(t) .\end{cases}
$$

The tumor surface evolves with normal velocity

$$
V=-n \cdot \nabla \bar{p}+G n \cdot \nabla \bar{\psi}-A G \frac{n \cdot x}{2} \quad \text { on } \partial \Omega(t) .
$$

Proof. From the relations (2.1), (2.3), (2.13) and (2.14) we find that

$$
\Delta \bar{\psi}=\frac{\lambda_{B}+\lambda}{D} \bar{\psi} \quad \text { in } \Omega(t) .
$$

The balance between diffusion and nutrient consumption $\left(L_{D}=1\right)$ yields the first equation of (2.16). Equation (2.14) further implies

$$
\bar{\psi}=\frac{\sigma-\sigma^{\infty} B}{\sigma^{\infty}(1-B)},
$$

and in view of (2.7) we obtain the following boundary condition on $\partial \Omega(t)$ :

$$
\bar{\psi}=\frac{\sigma^{\infty}-\sigma^{\infty} B}{\sigma^{\infty}-\sigma^{\infty} B}=1
$$

From the relations (2.4)-(2.6) we obtain that

$$
\Delta P=\frac{1}{\mu}\left(\lambda_{A}-b \sigma\right)
$$


Recalling $\bar{\psi}=\left(\sigma-\sigma^{\infty} B\right) /\left(\sigma^{\infty}(1-B)\right)$, the relations $(2.11),(2.12),(2.14)$, and (2.15) yield

$$
\Delta \bar{p}=\frac{1}{\mu \gamma}\left(\lambda_{A}-b \sigma\right)+\frac{\sigma-\sigma^{\infty} B}{\sigma^{\infty}(1-B)} \frac{\lambda_{M}}{\lambda_{R}}(1-B)-\frac{\lambda_{M}}{\lambda_{R}}(1-B) \frac{\frac{\lambda_{A}}{\lambda_{M}}-B}{1-B} .
$$

Furthermore (2.10) implies

$$
\Delta \bar{p}=\frac{\lambda_{A}}{\lambda_{R}}-\frac{\lambda_{A}}{\lambda_{R}}-\sigma\left(\frac{b}{\lambda_{R}}-\frac{\lambda_{M}}{\lambda_{R} \sigma^{\infty}}\right) .
$$

Since $b=\lambda_{M} / \sigma^{\infty}$ it follows that

$$
\Delta \bar{p}=0 \quad \text { in } \Omega(t) .
$$

The second equation of (2.17) follows now from (2.8) and (2.15).

Equation (2.18) follows straightforwardly from previous relations. This completes the proof.

\section{Radially symmetric stationary solutions}

Let $(A, G) \in \mathbb{R}^{2}$ be given. In the radially symmetric case, i.e. for $\Omega(t)=$ $D(0, R(t))$, system (1.1) reads as

$$
\left\{\begin{array}{lll}
\Delta \psi=f(\psi) & \text { in } D(0, R(t)), & t \geq 0, \\
\Delta p=0 & \text { in } D(0, R(t)), & t \geq 0, \\
\psi=1 & \text { on } \partial D(0, R(t)), & t \geq 0, \\
p=\frac{1}{R(t)}-A G \frac{R^{2}(t)}{4} & \text { on } \partial D(0, R(t)), & t \geq 0, \\
G \frac{\partial \psi}{\partial n}-\frac{\partial p}{\partial n}-A G \frac{R(t)}{2}=R^{\prime}(t) & \text { on } \partial D(0, R(t)), & t>0 \\
R(0)=R_{0}, & &
\end{array}\right.
$$

where $n=x /|x|$ and $R_{0}>0$. Notice that if $G=0$, then any disc is a stationary solution of (3.1). In the remaining of this section we consider therefore just the case $G \neq 0$.

Notice that the boundary conditions in (3.1) depend only on the radius of the tumor. This motivates us to look for solutions $(R, \psi, p) \in(0, \infty) \times$ $C^{2}([0, R]) \times C^{2}([0, R])$, solving the coupled problem

$$
\begin{cases}\psi^{\prime \prime}+\frac{1}{r} \psi^{\prime}=f(\psi) & 0<r<R, \\ p^{\prime \prime}+\frac{1}{r} p^{\prime}=0 & 0<r<R, \\ \psi(R)=1 & \\ p(R)=\frac{1}{R}-A G \frac{R^{2}}{4} & \\ G \psi^{\prime}(R)-p^{\prime}(R)-\frac{A G R}{2}=0 . & \end{cases}
$$

Problems (3.1) and (3.2) are equivalent in the following sens: if $(R, \psi, p)$ is a solution of $(3.2)$ then, letting $\Omega_{0}=D(0, R)$, the triple $\left(\Omega_{0}, \psi(|x|), p(|x|)\right)$ is a radially symmetric stationary solution of (3.1). Conversely, it is also immediate that any stationary solution of (3.1) determines a unique solution of (3.2). 


\subsection{The semilinear Dirichlet problem}

We prove first that, given $R>0$, the initial value problem

$$
\left\{\begin{array}{l}
\psi^{\prime \prime}+\frac{1}{r} \psi^{\prime}=f(\psi), \quad 0<r<R \\
\psi(R)=1
\end{array}\right.
$$

possesses a unique solution $\psi \in C^{2}([0, R])$. To this scope we shall use the so called shooting method. Suppose we have found a solution to (3.3). Multiplying with $r$ the differential equation of (3.3) we get that $\left(r \psi^{\prime}(r)\right)^{\prime}=r f(\psi(r))$ for $r \in[0, R]$. Integrating, we obtain

$$
\psi^{\prime}(r)=\frac{1}{r} \int_{0}^{r} s f(\psi(s)) \mathrm{d} s,
$$

thus we have $\psi^{\prime}(s)=0$. Therefore, given $c \in(0,1)$, consider the initial value problem

$$
\left\{\begin{array}{l}
\psi^{\prime \prime}+\frac{1}{r} \psi^{\prime}=f(\psi), \quad 0<r \\
\psi(0)=c \\
\psi^{\prime}(0)=0
\end{array}\right.
$$

Theorem 3.1. Given $c \in(0,1)$, there exists a unique maximal solution $\psi_{c} \in C^{\infty}$ $\left(\left[0, R_{c}^{*}\right)\right.$ of $(3.5)$ and

$$
\lim _{r \nearrow R_{c}^{*}} \psi_{c}(r)=\infty
$$

Proof. We prove first short time existence and uniqueness of the solution to (3.5). From (3.4) we obtain that any solution to (3.5) satisfies

$$
\psi(r)=c+\int_{0}^{r} \frac{1}{s} \int_{0}^{s} t f(\psi(t)) \mathrm{d} t \mathrm{~d} s, \quad r \geq 0 .
$$

Let $b>0, M:=\max _{[0, c+b]}\left(f+f^{\prime}\right)$ and $h:=\min \{\sqrt{b / M}, \sqrt{1 / 2 M}\}$. The set

$$
B:=\left\{u:[0, h] \rightarrow \mathbb{R}_{+} \mid u \text { is continuous, }\|u-c\|_{\infty} \leq b\right\}
$$

is a closed set in the Banach space $\left(C([0, h], \mathbb{R}),\|\cdot\|_{\infty}\right)$, thus is a complete metric space. The mapping $T: B \rightarrow B$, defined by

$$
T u(r):=c+\int_{0}^{r} \frac{1}{s} \int_{0}^{s} t f(u(t)) \mathrm{d} t \mathrm{~d} s, \quad \text { for } \quad r \geq 0 \text { and } u \in B,
$$

is $1 / 2$-contraction. Indeed, for $u \in B$ we have

$$
|T u(r)-c|=\int_{0}^{r} \frac{1}{s} \int_{0}^{s} t f(u(t)) \mathrm{d} t \mathrm{~d} s \leq \int_{0}^{r} \int_{0}^{s} f(u(t)) \mathrm{d} t \mathrm{~d} s \leq M h^{2} \leq b,
$$

for all $r \in[0, h]$, thus $T(B) \subset B$. Moreover, for $u, v \in B$ we have

$$
|T u(r)-T v(r)| \leq \int_{0}^{r} \frac{1}{s} \int_{0}^{s} t|f(u(t))-f(v(t))| \mathrm{d} t \mathrm{~d} s \leq M h^{2}\|u-v\|_{\infty},
$$

for all $r \in[0, h]$, hence $\|T u-T v\|_{\infty} \leq 1 / 2\|u-v\|_{\infty}$. The Banach fixed point theorem implies that $T$ has a unique fixed point $\psi_{c}$ in $B$, thus (3.5) has a unique solution in the set $C^{2}([0, h])$. 
Let $R_{c}^{*}$ denote the maximal existence time for this solution. It follows easily that $\psi_{c}$ is the unique solution to $(3.5)$ satisfying $\psi_{c}(0)=c$. Using an induction argument, we get that $\psi_{c} \in C^{\infty}\left(\left[0, R_{c}^{*}\right)\right)$.

We are left to prove relation (3.6). We first observe that $\psi_{c}$ and $\psi_{c}^{\prime}$ are both strictly increasing on $\left[0, R_{c}^{*}\right)$. Indeed, given $0<t<R_{c}^{*}$, we see that $\psi_{c}^{\prime}$ is a solution of the problem

$$
\left\{\begin{array}{l}
u^{\prime \prime}+\frac{1}{r} u^{\prime}-\left(\frac{1}{r^{2}}+f^{\prime}\left(\psi_{c}\right)\right) u=0, \quad 0<r<t, \\
u(0)=0
\end{array}\right.
$$

Because $\psi_{c}^{\prime}$ is positive and continuous on $[0, t]$, it must attain its maximum in $t$. Let $h$ be the constant found in the first part of the proof. The pair $\left(\psi_{c}, \psi_{c}^{\prime}\right)$ is the unique solution of the problem

$$
\begin{cases}(u, v)^{\prime}=g(r,(u, v)), & \frac{h}{2}<r<R_{c}^{*}, \\ (u, v)\left(\frac{h}{2}\right)=\left(\psi_{c}\left(\frac{h}{2}\right), \psi_{c}^{\prime}\left(\frac{h}{2}\right)\right), & \end{cases}
$$

with $g:(0, \infty) \times \mathbb{R}^{2} \rightarrow \mathbb{R}^{2}$ given by $g(r,(u, v))=(v,-v / r+f(u))$. We conclude that $R_{c}^{*}=\infty$ or $\left|\left(\psi_{c}, \psi_{c}^{\prime}\right)\right| \rightarrow \infty$ as $r \rightarrow R_{c}^{*}$.

Suppose first that $R_{c}^{*}=\infty$. Since $\psi_{c} \geq c$, we obtain for $r \geq 0$ that

$$
\psi_{c}^{\prime}(r)=\frac{1}{r} \int_{0}^{r} s f\left(\psi_{c}(s)\right) \mathrm{d} s \geq \frac{1}{r} \int_{0}^{r} s f(c) \mathrm{d} s \geq \frac{r^{2}}{2 r} f(c) \geq \frac{r}{2} f(c),
$$

A further integration shows that $\lim _{r \rightarrow R_{c}^{*}} \psi_{c}(r)=\infty$. In the other case we have either $\psi_{c} \rightarrow \infty$ or $\psi_{c}^{\prime} \rightarrow \infty$, and the conclusion holds.

Given $c \in(0,1)$, let $\psi_{c} \in C^{\infty}\left(\left[0, R_{c}^{*}\right)\right)$ be the solution of problem (3.5). There exists a unique $R_{c} \in\left(0, R_{c}^{*}\right)$ such that

$$
\psi_{c}\left(R_{c}\right)=1 \text {. }
$$

Further on we study the mapping $\left[c \mapsto R_{c}\right]$. Let $\mathcal{R}:(0,1) \longrightarrow \mathbb{R}_{+}$be given by $\mathcal{R}(c)=R_{c}$. We observe that for $c_{1}<c_{2}$ we have $\psi_{c_{1}}<\psi_{c_{2}}, R_{c_{1}}^{*} \geq R_{c_{2}}^{*}$ and $R_{c_{1}}>R_{c_{2}}$, i.e. $\mathcal{R}$ is a strictly decreasing function. As the next results show, the function $\mathcal{R}$ is continuous and bijective.

Lemma 3.2. Given $c_{0} \in(0,1)$ and $R<R_{c_{0}}^{*}$, we have

$$
\lim _{c \nearrow c_{0}[0, R]} \max _{c}\left|\psi_{c}-\psi_{c_{0}}\right|=0 \text {. }
$$

Proof. Since $c<c_{0}$ we have $R_{c}^{*} \geq R_{c_{0}}^{*}, \psi_{c} \in C^{\infty}\left(\left[0, R_{c_{0}}^{*}\right)\right)$ and $\psi_{c}<\psi_{c_{0}}$. Let $R<R_{c_{0}}^{*}$ be given and set $M:=\max _{\left[0, \psi_{c_{0}}(R)\right]} f^{\prime}$. Given $r \in[0, R]$, we get

$$
\begin{aligned}
\left|\psi_{c_{0}}(r)-\psi_{c}(r)\right| & \leq\left|c_{0}-c\right|+\int_{0}^{r} \frac{1}{t} \int_{0}^{t} s\left|f\left(\psi_{c_{0}}(s)\right)-f\left(\psi_{c}(s)\right)\right| \mathrm{d} s \mathrm{~d} t \\
& \leq\left|c_{0}-c\right|+\int_{0}^{r} \int_{0}^{t} M\left|\psi_{c_{0}}(s)-\psi_{c}(s)\right| \mathrm{d} s \mathrm{~d} t \\
& \leq\left|c_{0}-c\right|+M R \int_{0}^{r}\left|\psi_{c_{0}}(s)-\psi_{c}(s)\right| \mathrm{d} s
\end{aligned}
$$


Gronwall's lemma implies

$$
\left|\psi_{c_{0}}(r)-\psi_{c}(r)\right| \leq\left(c_{0}-c\right) e^{M R^{2}},
$$

which completes the proof.

As a direct consequence of the previous lemma we obtain:

Corollary 3.3. The function $\mathcal{R}$ is continuous from the left.

Proof. Let $c_{0} \in(0,1)$ be given and presuppose that $\lim _{c / c_{0}} \mathcal{R}(c)>\mathcal{R}\left(c_{0}\right)$. We find then a constant $b \in\left(\mathcal{R}\left(c_{0}\right), R_{c_{0}}^{*}\right)$ such that $\mathcal{R}\left(c_{0}\right)<b<\mathcal{R}(c)$ for all $c \in\left(0, c_{0}\right)$. Lemma 2.2 yields

$$
1 \geq \lim _{c \nearrow c_{0}} \psi_{c}(b)=\psi_{c_{0}}(b)>1
$$

and we are done.

Proposition 3.4. The function $\mathcal{R}$ is continuous.

Proof. We are left to prove that $\mathcal{R}$ is continuous from the right. We assume by contradiction that $\lim _{c \backslash c_{0}} \mathcal{R}(c)<\mathcal{R}\left(c_{0}\right)$. Given $c \in(0,1)$, let $\widetilde{R}_{c} \in(0, \infty)$ be defined as the point with $\psi_{c}\left(\widetilde{R}_{c}\right)=2$. The mapping $\left[c \mapsto \widetilde{R}_{c}\right]$ is also strictly decreasing. From (3.7) we obtain

$$
\psi_{c}\left(\widetilde{R}_{c}\right)-\psi_{c}\left(R_{c}\right)=\int_{R_{c}}^{\widetilde{R}_{c}} \frac{1}{r} \int_{0}^{r} t f\left(\psi_{c}(t)\right) \mathrm{d} t \mathrm{~d} r,
$$

hence

$$
\begin{aligned}
1 & \leq\left(\widetilde{R}_{c}-R_{c}\right) \max _{\left[0, \widetilde{R}_{c}\right]} \frac{1}{r} \int_{0}^{r} t f\left(\psi_{c}(t)\right) \mathrm{d} t \\
& \leq\left(\widetilde{R}_{c}-R_{c}\right) f(2) \widetilde{R}_{c_{0}}
\end{aligned}
$$

for all $c>c_{0}$. Thus, we get

$$
\lim _{c \searrow c_{0}} \widetilde{R}_{c} \geq \frac{1}{f(2) \widetilde{R}_{c_{0}}}+\lim _{c \searrow c_{0}} R_{c}
$$

Choose $a>0$ such that

$$
\min \left\{\frac{1}{f(2) \widetilde{R}_{c_{0}}}+\lim _{c \searrow c_{0}} R_{c}, \mathcal{R}\left(c_{0}\right)\right\}>a>\lim _{c \searrow c_{0}} R_{c} .
$$

Since $\lim _{c \backslash c_{0}} \widetilde{R}_{c}>a$ there exists $c_{1}>c_{0}$ with $\widetilde{R}_{c}>a$ if $c \in\left[c_{0}, c_{1}\right]$. Consequently, $[0, a] \subset\left[0, \widetilde{R}_{c}\right] \subset\left[0, R_{c}^{*}\right)$ for all $c_{0} \leq c \leq c_{1}$. Letting $M:=$ $\max _{\left[0, \psi_{c_{1}}(a)\right]} f^{\prime}$ we repeat the arguments presented in the proof of Lemma 3.2 to obtain

$$
\left|\psi_{c}(r)-\psi_{c_{0}}(r)\right| \leq M\left(c-c_{0}\right) e^{M a^{2}},
$$

for all $c \in\left[c_{0}, c_{1}\right]$ and $r \in[0, a]$. It follows that $\psi_{c}(a)-\psi_{c_{0}}(a) \rightarrow_{c \searrow c_{0}} 0$, and so

$$
1>\psi_{c_{0}}(a)=\lim _{c \searrow c_{0}} \psi_{c}(a) \geq 1 \text {. }
$$


This is a contradiction, thus our assumption was false, and the proof is complete.

Proposition 3.5. The mapping $\mathcal{R}:(0,1) \longrightarrow(0, \infty)$ is bijective. More precisely, we have:

$$
\lim _{c \nearrow 1} \mathcal{R}(c)=0 \quad \text { and } \quad \lim _{c \searrow 0} \mathcal{R}(c)=\infty .
$$

Proof. Let $c \in(0,1)$ be given. From (3.4) we obtain that $\psi_{c}^{\prime}(r) \geq f(1 / 2) r / 2$ for all $0 \leq r<R_{c}^{*}$, provided $c>1 / 2$. Integrating, we get

$$
1=\psi_{c}\left(R_{c}\right) \geq c+\frac{R_{c}^{2}}{4} f\left(\frac{1}{2}\right),
$$

and, letting $c \nearrow 1$, we obtain the first relation in (3.11).

We presuppose now that $\lim _{c \searrow 0} R_{c}=: T<\infty$. Following the lines in the proof of Proposition 3.4 we have

$$
\psi_{c}\left(\widetilde{R}_{c}\right)-\psi_{c}\left(R_{c}\right) \leq\left(\widetilde{R}_{c}-R_{c}\right) \widetilde{R}_{c} f(2) .
$$

Integrating the relation $\left(r \psi_{c}^{\prime}(r)\right)^{\prime}=r f\left(\psi_{c}(r)\right)$, we obtain

$$
\psi_{c}^{\prime}(r)=\frac{R_{c} \psi_{c}^{\prime}\left(R_{c}\right)}{r}+\frac{1}{r} \int_{R_{c}}^{r} s f\left(\psi_{c}(s)\right) \mathrm{d} s
$$

for all $R_{c} \leq r<R_{c}^{*}$. Thus

$$
\begin{aligned}
1=\psi_{c}\left(\widetilde{R}_{c}\right)-\psi_{c}\left(R_{c}\right) & =R_{c} \psi_{c}^{\prime}\left(R_{c}\right) \int_{R_{c}}^{\widetilde{R}_{c}} \frac{1}{r} \mathrm{~d} r+\int_{R_{c}}^{\widetilde{R}_{c}} \frac{1}{r} \int_{R_{c}}^{r} s f\left(\psi_{c}(s)\right) \mathrm{d} s \mathrm{~d} r \\
& \geq f(1)\left(\widetilde{R}_{c}-R_{c}\right)^{2} / 4
\end{aligned}
$$

for all $c \in(0,1)$. Consequently,

$$
\widetilde{R}_{c} \leq \frac{2}{\sqrt{f(1)}}+R_{c} \leq \frac{2}{\sqrt{f(1)}}+T
$$

The relations (3.12) and (3.13) enforce

$$
\widetilde{R}_{c} \geq R_{c}+\frac{1}{f(2)\left(\frac{2}{\sqrt{f(1)}}+T\right)}
$$

Since $R_{c} \nearrow T$ for $c \searrow 0$, there exists $c_{0} \in(0,1)$ with $\widetilde{R}_{c}>T$ for all $c \in\left(0, c_{0}\right]$, and so $[0, T] \subset\left[0, R_{c}^{*}\right)$ for $c \in\left(0, c_{0}\right]$. Further on, we have $\psi_{c}(r) \leq \psi_{c_{0}}(r) \leq$ $\psi_{c_{0}}(T)$ for all $r \in[0, T]$ and $c \in\left(0, c_{0}\right]$. With $M:=\max _{\left[0, \psi_{c_{0}}(T)\right]} f^{\prime}$ we obtain

$$
\psi_{c}^{\prime}(r) \leq \int_{0}^{r}\left(f\left(\psi_{c}(\tau)\right)-f(0)\right) \mathrm{d} \tau \leq M \int_{0}^{r} \psi_{c}(\tau) \mathrm{d} \tau,
$$

for all $r \in[0, T]$ and $c \in\left(0, c_{0}\right]$. Integrating and using Fubini's theorem we get

$$
\psi_{c}(r) \leq c+M T \int_{0}^{r} \psi_{c}(s) \mathrm{d} s
$$

for $r \in[0, T]$ and $c \leq c_{0}$. Gronwall's lemma implies

$$
\left\|\psi_{c}\right\|_{\infty,[0, T]} \leq c e^{M T^{2}}, \quad \forall c \in\left(0, c_{0}\right]
$$


Letting $c \searrow 0$ in relation $(3.14)$, we conclude that $\left\|\psi_{c}\right\|_{\infty,[0, T]} \longrightarrow 0$, in contradiction with $R_{c}<T$ and $\psi_{c}\left(R_{c}\right)=1$. Thus, our assumption was false and this completes the proof.

Theorem 3.6. Given $R>0$, there exists a unique solution $\psi_{R} \in C^{\infty}\left(\left[0, R^{*}\right)\right.$, $R<R^{*}$, to problem (3.3).

Proof. Let $R>0$ be given. From Propositions 3.4 and 3.5 we conclude that there exists a unique $c_{0} \in(0,1)$ such that $R_{c_{0}}=R$. Thus, $\psi_{R}:=\psi_{c_{0}}$ is a solution of (3.3).

We are left to prove that this solution is unique. Let $\psi_{R}(x)=\psi_{R}(|x|)$, $x \in \bar{D}(0,1)$. The function $\psi_{R}$ is then solution of

$$
\left\{\begin{array}{lll}
\Delta \psi=f(\psi) & \text { in } & D(0, R), \\
\psi=1 & \text { on } & \partial D(0, R) .
\end{array}\right.
$$

Let $\varphi$ be another solution of (3.15). Then $u=\psi_{R}-\varphi \in C^{2}(\bar{D}(0, R))$ must solve the Dirichlet problem

$$
\begin{cases}\Delta u=g u & \text { in } \quad D(0, R), \\ u=0 & \text { on } \quad \partial D(0, R),\end{cases}
$$

with $g(x)=\int_{0}^{1} f^{\prime}(t \psi(x)+(1-t) \varphi(x)) \mathrm{d} x$ for all $x \in D(0, R)$. From (1.2) we have $g>0$ which, together with the weak maximum principle, implies $u=0$ and we are done.

Lemma 3.7. The problem

$$
\left\{\begin{array}{l}
p^{\prime \prime}+\frac{1}{r} p^{\prime}=0, \\
p(R)=\frac{1}{R}-A G \frac{R^{2}}{4},
\end{array}\right.
$$

possesses a unique solution $p_{R} \in C^{\infty}([0, \infty))$, given by

$$
p_{R}(r)=\frac{1}{R}-A G \frac{R^{2}}{4}
$$

for all $r \in[0, \infty)$.

Summarizing, we have reduced the existence problem for (3.2) to an equation on the real line. More precisely, from Theorem 3.6 and Lemma 3.7 we get that problem (3.2) has solutions iff the equation

$$
A=\frac{2 \psi_{R}^{\prime}(R)}{R}
$$

is solvable. However, it is difficult to treat (3.18) directly. We shall first derive a suitable representation of $\psi_{R}$. 


\subsection{A representation of $\psi_{R}$}

In the following we consider the parameter-depending problem:

$$
\left\{\begin{array}{l}
\frac{\partial^{2} U}{\partial r^{2}}(r, \lambda)+\frac{1}{r} \frac{\partial U}{\partial r}(r, \lambda)=\lambda f(U(r, \lambda)), \quad 0 \leq r \leq 1 \\
\frac{\partial U}{\partial r}(0, \lambda)=0 \\
U(1, \lambda)=1
\end{array}\right.
$$

with $\lambda$ in $[0, \infty)$. As in the previous subsection, we can show that for each $\lambda \in[0, \infty)$ there exists a unique, non-decreasing solution $U(\cdot, \lambda) \in C^{\infty}([0,1])$ of (3.19).

We prove now that the Fréchet derivative of $U$ is uniformly Lipschitzcontinuous on compact subsets of $[0,1] \times[0, \infty)$ and that, for fixed $r \in[0,1]$, the partial function $U(r, \cdot)$ is non-increasing on $[0, \infty)$.

Lemma 3.8. Given $r \in[0,1]$, the mapping $[0, \infty) \ni \lambda \mapsto U(r, \lambda)$ is decreasing.

Proof. If $\lambda=0$, then $U(r, 0)=1$ for all $r \in[0,1]$. Let $0<\lambda_{1}<\lambda_{2}, U_{1}:=$ $U\left(\cdot, \lambda_{1}\right), U_{2}:=U\left(\cdot, \lambda_{2}\right)$ and suppose that $c_{1}=U_{1}(0)<U_{2}(0)=c_{2}$, with $c_{1}, c_{2} \in(0,1)$. Then, since $U_{1}(1)=U_{2}(1)=1$, we find $\bar{r}$ with $U_{1}(\bar{r})=U_{2}(\bar{r})$ and $U_{1}<U_{2}$ on $[0, \bar{r}]$. For $t \in[0, \bar{r}]$ we have

$$
\begin{aligned}
U_{2}^{\prime}(t)-U_{1}^{\prime}(t) & =\frac{\lambda_{2}}{t} \int_{0}^{t} s f\left(U_{2}(s)\right) \mathrm{d} s-\frac{\lambda_{1}}{t} \int_{0}^{t} s f\left(U_{1}(s)\right) \mathrm{d} s \\
& \geq \frac{\lambda_{2}}{t} \int_{0}^{t} s\left(f\left(U_{2}(s)\right)-f\left(U_{1}(s)\right)\right) \mathrm{d} s \geq 0 .
\end{aligned}
$$

Integrating, we obtain $U_{2}(t)-U_{1}(t) \geq c_{2}-c_{1}>0$ on $[0, \bar{r}]$ and that is a contradiction, so $c_{2} \leq c_{1}$.

Suppose now that $c_{2}=c_{1}$. Then we obtain

$$
\begin{aligned}
U_{2}^{\prime \prime}-U_{1}^{\prime \prime}+\frac{1}{r}\left(U_{2}^{\prime}-U_{1}^{\prime}\right) & =\lambda_{2} f\left(U_{2}\right)-\lambda_{1} f\left(U_{1}\right) \\
& =\lambda_{2}\left(f\left(U_{2}\right)-f\left(U_{1}\right)\right)+\left(\lambda_{2}-\lambda_{1}\right) f\left(U_{1}\right),
\end{aligned}
$$

and, using the mean value theorem, we get, for $t \in[0,1]$ that

$$
f\left(U_{2}(t)\right)-f\left(U_{1}(t)\right)=\int_{0}^{1} f^{\prime}\left(U_{1}(t)+s\left(U_{2}(t)-U_{1}(t)\right)\right) \cdot\left(U_{2}(t)-U_{1}(t)\right) \mathrm{d} s .
$$

Letting $W=U_{2}-U_{1}$ we have

$$
\left\{\begin{array}{l}
W^{\prime \prime}+\frac{1}{t} W^{\prime}-g W>0, \quad \text { in }(0,1) \\
W(0)=W(1)=0
\end{array}\right.
$$


with a smooth, positive function $g$. Therefore $W<0$ in $(0,1)$. Further on, we have

$$
\begin{aligned}
\lim _{t \rightarrow 0}\left(U_{2}^{\prime \prime}(t)-U_{1}^{\prime \prime}(t)\right)=\lim _{t \rightarrow 0}[ & -\frac{\lambda_{2}}{t^{2}} \int_{0}^{t} s f\left(U_{2}(s)\right) \mathrm{d} s \\
& \left.\quad+\frac{\lambda_{1}}{t^{2}} \int_{0}^{t} s f\left(U_{1}(s)\right) \mathrm{d} s+\lambda_{2}\left(f\left(U_{2}\right)\right)-\lambda_{1}\left(f\left(U_{1}\right)\right)\right] \\
= & \frac{\lambda_{2}-\lambda_{1}}{2} f\left(c_{2}\right)>0 .
\end{aligned}
$$

Hence, we find $\delta \in(0,1)$ such that

$$
U_{2}(t)-U_{1}(t) \geq \int_{0}^{t} \frac{\lambda_{2}-\lambda_{1}}{4} f\left(c_{2}\right) s \mathrm{~d} s>0, \quad \forall t \in[0, \delta],
$$

in contradiction with $U_{2}<U_{1}$ on $(0,1)$. Thus, $c_{2}<c_{1}$, for $\lambda_{1}<\lambda_{2}$. The weak maximum principle implies $U_{2}<U_{1}$ in $[0,1)$.

We need also the following theorem:

Theorem 3.9. Let $X, Y, Z$ be metric spaces. Then $f: X \times Y \longrightarrow Z$ is uniformly Lipschitz-continuous iff $f$ is uniformly Lipschitz-continuous with respect to both variables.

Proof. See the proof of Lemma 8.1 in [1].

Lemma 3.10. $U$ is uniformly Lipschitz-continuous on $[0,1] \times[0, N]$, for all $N \in(0, \infty)$.

Proof. Let $N>0$ be fixed. For $(x, \lambda) \in[0,1] \times[0, \infty)$ we obtain from (3.19) that

$$
U(x, \lambda)=1-\int_{0}^{1} \frac{1}{r} \int_{0}^{r} s \lambda f(U(s, \lambda)) \mathrm{d} s \mathrm{~d} r+\int_{0}^{x} \frac{1}{r} \int_{0}^{r} s \lambda f(U(s, \lambda)) \mathrm{d} s \mathrm{~d} t .
$$

Relation (3.21) implies that $[0,1] \ni r \mapsto U(r, \lambda)$ is uniformly Lipschitz-continuous with respect to $\lambda \in[0, N]$.

For $0 \leq \lambda<\mu$ we have $U(\cdot, \lambda) \geq U(\cdot, \mu)$. It follows

$$
0<U(0, \lambda)-U(0, \mu) \leq \int_{0}^{1} \frac{1}{r} \int_{0}^{t} s(\mu-\lambda) f(1) \mathrm{d} s \mathrm{~d} r \leq f(1)(\mu-\lambda) .
$$

Thus,

$$
|U(0, \lambda)-U(0, \mu)| \leq f(1)|\mu-\lambda| .
$$

From (3.21), for $\lambda, \mu \geq 0$ and letting $M=\max _{[0,1]} f^{\prime}>0$, we obtain, using Fubini's theorem that

$$
|U(x, \lambda)-U(x, \mu)| \leq 2 f(1)|\mu-\lambda|+N M \int_{0}^{x}|U(s, \lambda)-U(s, \mu)| \mathrm{d} s .
$$

The conclusion follows using Gronwall's lemma and Theorem 3.9.

From Lemma 3.10 we obtain the following result:

Lemma 3.11. The partial derivative of $U$ with respect to $r$ is uniformly Lipschitz-continuous on $[0,1] \times[0, N]$, for any fixed $N \in(0, \infty)$. 
Proof. Let $N \in(0, \infty)$ be fixed and $V(r, \lambda):=\partial U / \partial r(r, \lambda)$. We have

$$
\frac{V(r, \lambda)}{r}=\frac{1}{r^{2}} \int_{0}^{r} s \lambda f(U(s, \lambda)) \mathrm{d} s \leq \frac{\lambda}{2} f(1),
$$

for all $r \in[0,1]$, and together with (3.19) we obtain that

$$
\left|\frac{\partial V}{\partial r}(r, \lambda)\right| \leq \frac{3}{2} \lambda f(1) \leq \frac{3}{2} N f(1):=L, \quad \forall(r, \lambda) \in[0,1] \times[0, N] .
$$

Consequently, $|V(r, \lambda)-V(s, \lambda)| \leq L|r-s|$, thus $[0,1] \ni r \mapsto V(r, \lambda)$ is uniformly Lipschitz-continuous with respect to $\lambda$.

For $r \in(0,1]$ and $\lambda, \mu \geq 0$ we have

$$
|V(r, \lambda)-V(r, \mu)| \leq\left(f(1)+N M L_{N}\right)|\lambda-\mu|,
$$

where $L_{N}$ is the Lipschitz constant for $U$ on $[0,1] \times[0, N]$. Using the continuity of $V$ with respect to $r$ and applying once again Theorem 3.9 we obtain the desired result.

Let us now study the differentiability of $U$ with respect to the variable $\lambda$. Given $\lambda \geq 0$, we consider the following Dirichlet problem

$$
\begin{cases}\Delta v(x, \lambda)-\lambda f^{\prime}(U(x, \lambda)) v(x, \lambda)=f(U(x, \lambda)), & \text { in } \quad D(0,1) \\ v(x, \lambda)=0, & \text { on } \quad \partial D(0,1),\end{cases}
$$

where $U(x, \lambda):=U(|x|, \lambda)$ is the solution of the Dirichlet problem

$$
\begin{cases}\Delta U(x, \lambda)=\lambda f(U(x, \lambda)), & \text { in } \quad D(0,1), \\ U(x, \lambda)=1, & \text { on } \quad \partial D(0,1) .\end{cases}
$$

Problem (3.23) has a unique solution $v \in C^{\infty}(\bar{D}(0,1))$, and using the estimate (3.12) in [10], we find a positive constant $c$, depending only on $f$, such that

$$
\|v(\cdot, \lambda)\|_{\infty, \bar{D}(0,1)} \leq c\|f(U(\cdot, \lambda))\|_{\infty, \bar{D}(0,1)} \leq c f(1), \quad \forall \lambda \geq 0 .
$$

Let now $N>0$ and $\lambda \in[0, N]$ be fixed. Let further $\mu \in[0,1]$, if $\lambda=0$, respectively $\mu \in[-\lambda / 2,1 / 2]$, if $\lambda \in(0, N]$. We set

$$
\omega(x):=U(x, \lambda+\mu)-U(x, \lambda)-\mu v(x, \lambda),
$$

for all $|x| \leq 1$. Then $\omega \in C^{\infty}(\bar{D}(0,1))$ is a solution of the Dirichlet problem

$$
\begin{cases}\Delta \omega(x)-\lambda f^{\prime}(U(x, \lambda) \omega(x)=h(x), & \text { in } D(0,1), \\ \omega(x)=0, & \text { on } \partial D(0,1),\end{cases}
$$

where $h \in C^{\infty}(\bar{D}(0,1))$ satisfies $|h(x)| \leq C|\mu|^{2}$, for all $x$ in $\bar{D}(0,1)$ and $\mu$ in the appropriate set, with a constant $C$ independent of $\mu$. Applying again estimate (3.12) in [10] we obtain

$$
\|\omega\|_{\infty, \bar{D}(0,1)} \leq c|\mu|^{2} .
$$

Particularly, $U$ is differentiable on $D(0,1) \times[0, \infty)$ with respect to the variable $\lambda$ and $\partial U / \partial \lambda=v$.

Lemma 3.12. Given $N \in(0, \infty)$, the mapping $[0, N] \ni \lambda \mapsto v(x, \lambda)$ is uniformly Lipschitz-continuous with respect to the variable $x \in D(0,1)$. 
Proof. Let $\lambda, \mu \in[0, N]$ be given and set $w(x)=v(x, \lambda)-v(x, \mu)$, for $x$ in $\bar{D}(0,1)$. Since $w$ is a solution of the Dirichlet problem

$$
\left\{\begin{array}{rlrl}
\Delta w-\lambda f^{\prime}(U(\cdot, \lambda)) w= & f(U(\cdot, \lambda))-f(U(\cdot, \mu))+v(\cdot, \mu)\left[\lambda f^{\prime}(U(\cdot, \lambda))\right. \\
& -\mu f^{\prime}(U(\cdot, \mu)], & & \text { in } D(0,1), \\
w= & 0, & & \text { on } \partial D(0,1),
\end{array}\right.
$$

we get that $\|\omega\|_{\infty, \bar{D}(0,1)} \leq c|\mu-\lambda|$, with a constant $c$ depending only on $f$ and $N$, cf. (3.25). This completes the proof.

Given $t \in[0,1], x \in \bar{D}(0,1)$ with $t=|x|$ and $\lambda \in[0, \infty)$, we have

$$
\begin{aligned}
\lim _{\mu \rightarrow 0} \frac{U(t, \lambda+\mu)-U(t, \lambda)}{\mu} & =\lim _{\mu \rightarrow 0} \frac{U(|x|, \lambda+\mu)-U(|x|, \lambda)}{\mu} \\
& =\lim _{\mu \rightarrow 0} \frac{U(x, \lambda+\mu)-U(x, \lambda)}{\mu}=v(x, \lambda),
\end{aligned}
$$

thus $v$ is radially symmetric. Lemma 3.12 implies that $U$ is differentiable on $[0,1] \times[0, \infty)$ with respect to $\lambda$ and that $\partial U / \partial \lambda=v$. Of course we consider the restriction of $v$ to the unit interval $[0,1]$ here.

We still have to show that $v$ is uniformly Lipschitz-continuous on $[0,1] \times$ $[0, N], N>0$, with respect to $r$. Therefore, it is enough to prove that $\partial v / \partial r$ is uniformly bounded on $[0,1] \times[0, N]$. Indeed, from $(3.23)$, in view of the radial symmetry, $v$ is the solution to the problem

$$
\begin{cases}\frac{\partial^{2} v}{\partial r^{2}}(r, \lambda)+\frac{1}{r} \frac{\partial v}{\partial r}(x, \lambda)-\lambda f^{\prime}(U(r, \lambda)) v(r, \lambda)=f(U(r, \lambda)), & 0<r<1 \\ v(r, \lambda)=0, & r=1 .\end{cases}
$$

We then obtain

$$
\frac{\partial v}{\partial r}(r, \lambda)=\frac{1}{r} \int_{0}^{r} s\left[\lambda f^{\prime}(U(s, \lambda)) v(s, \lambda)+f(U(s, \lambda))\right] \mathrm{d} s,
$$

and the uniform boundedness of $\partial v / \partial r$ on $[0,1] \times[0, N]$ follows in virtue of $(3.25)$.

From the Lemmas 3.10-3.12 and Theorem 3.9 we obtain the following Theorem

Theorem 3.13. The mapping $U$ is continuously differentiable on $[0,1] \times[0, \infty)$ with uniformly Lipschitz-continuous derivatives on compact subsets of $[0,1] \times$ $[0, \infty)$.

The function $U$ will play an important role in our analysis. First it will help us to determine the solution of (3.3), as the next theorem shows.

Theorem 3.14. Given $R>0$ we denote by $\psi_{R}$ the solution to (3.3). We have

$$
\psi_{R}(r)=U\left(\frac{r}{R}, R^{2}\right), \quad 0 \leq r \leq R .
$$

Proof. The proof follows by direct computations. 
Proof of Theorem 1.1. From Theorems 3.6, 3.14, Lemma 3.7 and relation (3.18) we conclude that (3.2) has a solution iff there exists a positive $R$ solving the equation

$$
A=\frac{2}{R^{2}} \frac{\partial U}{\partial r}\left(1, R^{2}\right)=2 \int_{0}^{1} r f\left(U\left(r, R^{2}\right)\right) \mathrm{d} r .
$$

We define the function

$$
F:[0, \infty) \rightarrow \mathbb{R}, \quad F(R)=2 \int_{0}^{1} r f\left(U\left(r, R^{2}\right)\right) \mathrm{d} r .
$$

From Lemma 3.8 and Theorem 3.13 we get that $F$ is continuously differentiable and strictly decreasing on $[0, \infty)$, with $F(0)=f(1)$.

Moreover, we state that $\lim _{R \rightarrow \infty} F(R)=0$. If we had that $U(\cdot, \lambda) \rightarrow_{\lambda \rightarrow \infty}$ 0 a.e. on $[0,1]$, then this would follow by Lebesgue's dominated convergence theorem, since $U$ is bounded by 1 . Let us thus presuppose that this is not the case, i.e. there exists $r_{0} \in(0,1)$ such that

$$
U\left(r_{0}, \lambda\right) \geq a, \quad \forall \lambda \geq 0,
$$

with a positive constant $a$. It follows then

$$
\begin{aligned}
1 & \geq U(1, \lambda)-U\left(r_{0}, \lambda\right) \geq \lambda \int_{r_{0}}^{1} \frac{1}{r} \int_{r_{0}}^{r} s f(U(s, \lambda)) \mathrm{d} s \mathrm{~d} r \\
& \geq \lambda \int_{r_{0}}^{1} \frac{r_{0}}{r} \int_{r_{0}}^{r} f(a) \mathrm{d} s \mathrm{~d} r \geq \lambda r_{0} f(a) \frac{\left(1-r_{0}\right)^{2}}{2} \underset{\lambda \rightarrow \infty}{\longrightarrow} \infty .
\end{aligned}
$$

This is a contradiction, thus $U(\cdot, \lambda) \rightarrow_{\lambda \rightarrow \infty} 0$ a.e. on $[0,1]$.

Summarizing, $F([0, \infty))=(0, f(1)]$, hence $(3.27)$ has a positive solution iff $A \in(0, f(1))$. Given $A \in(0, f(1))$, let $R_{A}$ denote the unique solution to (3.27). Lemma 3.8 implies then that $(0, f(1)) \ni A \mapsto R_{A}$ is strictly decreasing. This completes the proof.

\section{Radially symmetric evolution of tumors}

In this section we aim to prove that problem (3.1) possesses for any initial data $R_{0}>0$ a unique, globally defined solution. A triple $\left(R(\cdot), \psi_{R(\cdot)}, p_{R(\cdot)}\right)$ is called solution to (3.1) if

$$
\begin{aligned}
R & \in C^{1}([0, \infty),(0, \infty)), \\
\left(\psi_{R(t)}, p_{R(t)}\right) & \in C^{2}([0, R(t)]), \quad \text { for } t \geq 0
\end{aligned}
$$

and if $\left(R(\cdot), \psi_{R(\cdot)}, p_{R(\cdot)}\right)$ satisfies the equations in (3.1) pointwise.

From Theorem 3.6 and Lemma 3.7 we deduce that $\left(R(\cdot), \psi_{R(\cdot)}, p_{R(\cdot)}\right)$ is a solution of the evolution problem (3.1) iff the function $R$ solves the following initial value problem

$$
\left\{\begin{array}{l}
R^{\prime}=h(R), \quad 0<t<T \\
R(0)=R_{0}
\end{array}\right.
$$


where $R_{0}>0$ is the radius describing the initial state of the tumor and

$$
h(R):=G R\left(\int_{0}^{1} r f\left(U\left(r, R^{2}\right)\right) \mathrm{d} r-\frac{A}{2}\right)=\frac{G R}{2}(F(R)-A), \quad R \geq 0 .
$$

The function $F$ is defined by (3.28).

Proof of Theorem 1.2. Let $(A, G) \in \mathbb{R}^{2}$ be fixed. The function $h$ is continuously differentiable and, since $U \leq 1$, has linear growth. For $R_{0}>0$ fixed, we define $R$ to be the maximal defined solution of (4.1). Let $T$ denote the maximal existence time of this solution. It follows that the solution $R$ is bounded on bounded intervals (see [1, Theorem 7.8]). If $G=0$, then $R=R_{0}$ on $[0, \infty)$.

We consider now the case $G \neq 0$. We split our argumentation as follows:

(a) Let $(A, G) \in(0, f(1)) \times(0, \infty)$. In this case 0 and $R_{A}$ are the only stationary solutions of (4.1). Since $h>0$ on $\left(0, R_{A}\right)$ and $h<0$ on $\left(R_{A}, \infty\right)$ it must hold $T=\infty$ and $R(t) \rightarrow R_{A}$ as $t \rightarrow \infty$. Moreover,

$h^{\prime}\left(R_{A}\right)=\frac{G R_{A}}{2} F^{\prime}\left(R_{A}\right)=2 G R_{A}^{2} \int_{0}^{1} r f\left(U\left(r, R_{A}^{2}\right)\right) \frac{\partial U}{\partial \lambda}\left(r, R_{A}^{2}\right) \mathrm{d} r:=-\omega<0$, hence $R_{A}$ is exponentially stable.

(b) Let $(A, G) \in[f(1), \infty) \times(0, \infty)$. In this case $R=0$ is the only zero of $h$ and $h<0$ on $(0, \infty)$. It follows then $T=\infty$ and $\lim _{t \rightarrow \infty} R(t)=0$. Moreover,

$$
\frac{R^{\prime}}{R}<\frac{G}{2}(f(1)-A)<0,
$$

which implies that $R(t)$ converges exponentially for $A>f(1)$.

(c) Let $(A, G) \in[f(1), \infty) \times(-\infty, 0)$. Since $G>0$ we have in this situation that $h>0$ on $(0, \infty)$. It follows that $R(t) \rightarrow \infty$ for $t \rightarrow T$. Because $R$ must be bounded on bounded intervals it must be $T=\infty$. That the convergence is at an exponential rate follows from the following inequality

$$
\frac{R^{\prime}}{R} \geq \frac{G}{2}\left(F\left(R_{0}\right)-A\right)
$$

(d) Let $(A, G) \in(0, f(1)) \times(-\infty, 0)$. As in (a), 0 and $R_{A}$ are the only two zeros of $h$, but now $h<0$ on $\left(0, R_{A}\right)$ and $h>0$ on $\left(R_{A}, \infty\right)$. If $R_{0}<R_{A}$, then, as in (b), $R$ decreases in infinite time to 0 . In this case the exponential convergence is implied by

$$
\frac{G}{2}(F(R)-A)<\frac{G}{2}\left(F\left(R_{0}\right)-A\right)<0 .
$$

If $R_{0}>R_{A}$ then we have the situation from (c), i.e. $\lim _{t \rightarrow \infty} R(t)=\infty$. Since

$$
\frac{G}{2}(F(R)-A)>\frac{G}{2}\left(F\left(R_{0}\right)-A\right)>0,
$$

the $R(t)$ converges exponentially fast. 
(e) In the situation considered here, $h$ and $G$ have the same sign. The radius $R(t)$ increases to infinity if $G>0$ and shrinks to zero for negative $G$. Moreover, we have that

$$
\begin{gathered}
\frac{R^{\prime}}{R} \geq-\frac{A G}{2}, \quad \text { if } \quad G>0 \\
\frac{R^{\prime}}{R} \leq \frac{G}{2}\left(F\left(R_{0}\right)-A\right), \quad \text { if } \quad G<0,
\end{gathered}
$$

on $[0, \infty)$. The desired result follows in view of the above relations.

\section{Acknowledgements}

The authors are grateful to the anonymous referee for several suggestions which improved the quality of our paper.

\section{References}

[1] Amann, H.: Ordinary Differential Equations. An Introduction to Nonlinear Analysis. Walter de Gruyter, Berlin (1990)

[2] Bellomo, N., Li, N.K., Maini, P.K.: On the foundations of cancer modelling: selected topics, speculations, and perspectives. Math. Models Methods Appl. Sci. 18(4), 593-647 (2008)

[3] Borisovich, A., Friedman, A.: Symmetric-breaking bifurcation for free boundary problems. Indiana Univ. Math. J. 54, 927-947 (2005)

[4] Byrne, H.M., Chaplain, M.A.: Growth of nonnecrotic tumors in the presence and absence of inhibitors. Math. Biosci. 130, 151-181 (1995)

[5] Cristini, V., Lowengrub, J., Nie, Q.: Nonlinear simulation of tumor growth. J. Math. Biol. 46, 191-224 (2003)

[6] Cui, S.B.: Analysis of a free boundary problem modeling tumor growth. Acta Math. Sin., English Ser. 21(5), 1071-1082 (2005)

[7] Cui, S.B., Escher, J.: Bifurcation analysis of an elliptic free boundary problem modelling the growth of avascular tumors. SIAM J. Math. Anal. 39(1), 210$235(2007)$

[8] Friedman, A., Reitich, F.: Analysis of a mathematical model for the growth of tumors. J. Math. Biol. 38, 262-284 (1999)

[9] Friedman, A., Reitich, F.: Symmetry-breaking bifurcation of analytic solutions to free boundary problems. Trans. Am. Math. Soc. 353, 1587-1634 (2001)

[10] Gilbarg, D., Trudinger, T.S.: Elliptic Partial Differential Equations of Secound Order. Springer, New York (1997)

[11] Greenspan, F.P.: On the growth and stability of cell cultures and solid tumors. J. Theor. Biol. 56, 229-242 (1976) 
J. Escher, A.-V. Matioc

Institut für Angewandte Mathematik,

Leibniz Universität Hannover, Welfengarten 1,

30167 Hannover, Germany

e-mail: matioca@ifam.uni-hannover.de

J. Escher

e-mail: escher@ifam.uni-hannover.de

Received: 28 January 2009.

Accepted: 2 July 2009. 